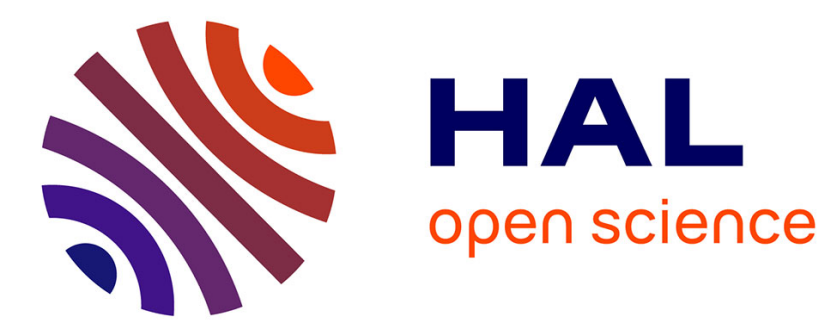

\title{
La transparence de l'atmosphere. VI. La brume blanche
} J. Duclaux

\section{To cite this version:}

J. Duclaux. La transparence de l'atmosphere. VI. La brume blanche. J. Phys. Radium, 1940, 1 (2), pp.41-43. 10.1051/jphysrad:019400010204100 . jpa-00233720

\section{HAL Id: jpa-00233720 https://hal.science/jpa-00233720}

Submitted on 1 Jan 1940

HAL is a multi-disciplinary open access archive for the deposit and dissemination of scientific research documents, whether they are published or not. The documents may come from teaching and research institutions in France or abroad, or from public or private research centers.
L'archive ouverte pluridisciplinaire HAL, est destinée au dépôt et à la diffusion de documents scientifiques de niveau recherche, publiés ou non, émanant des établissements d'enseignement et de recherche français ou étrangers, des laboratoires publics ou privés. 


\title{
LE JOURNAL DE PHYSIQUE
}

\author{
ET
}

\section{LE RADIUM}

\section{LA TRANSPARENGE DE L'ATMOSPHĖRE. VI. LA BRUME BLANGHE}

\author{
Par M. J. DUCLAUX.
}

\begin{abstract}
Sommaire. - On a souvent interprété les mesures de l'absorption atmosphérique en représentant les coefficients d'absorption par la somme de deux termes, l'un de diffusion moléculaire, l'autre indépendant de la longueur d'onde. Ce second terme correspondrait à une brume blanche, faisant disparaître les objets sans modifier leur couleur. Cette brume blanche ne peut être qu'exceptionnelle; dans l'immense majorité des cas, les effets obtenus par les filtres colorés en photographie, et l'observation visuelle, montrent que la brume absorbe les rayons d'autant plus que leur longueur d'onde est plus courte. Des mesures faites spécialement conduisent au même résultat. La décomposition de l'absorption en un terme de diffusion et un terme de brume blanche est donc un artifice qui ne peut donner que des résultats sans valeur.
\end{abstract}

1. - Les mesures du coefficient d'absorption $k$ de l'atmosphère ont été très souvent représentées par une formule du type

$$
k=a+b \lambda-m,
$$

dans laquelle $b \lambda^{-m}$ est le terme correspondant à la diffusion moléculaire, tandis que $a$ est un terme indépendant de la longueur d'onde, correspondant à ce que j'appellerai une brume blanche. Ce mode de décomposition de l'absorption totale revient à admettre qu'il n'y a que deux modes d'affaiblissement de la lumière : la diffusion moléculaire et la brume blanche, à l'exclusion de toute brume bleue, c'est-à-dire absorbant les rayons d'autant plus fortement que leur longueur d'onde est plus courte.

Ce mode de décomposition est purement artificiel et soulève, dès qu'on veut lui donner une application générale, de graves objections [ [ , 2]. Des observations très simples montrent en effet, de la manière la plus évidente, que la brume blanche, si elle existe, ne peut être qu'exceptionnelle, et ne peut par suite intervenir qu'exceptionnellement dans l'interprétation des mesures. Dans la très grande majorité des cas, la brume (en désignant par ce mot, opposé au mot brouillard, ce qui limite la visibilité à des distances de l'ordre du kilomètre ou davantage) a un pouvoir absorbant d'autant plus grand que la longueur d'onde est plus faible.

Si la brume blanche existait seule, avec une densité variable d'un jour à l'autre, le Soleil aurait, à une même hauteur au-dessus de l'horizon, toujours la même couleur, avec une brillance variable. L'obser- vation montre au contraire qu'à une petite hauteur le Soleil peut avoir toutes les colorations entre le blanc et le rouge.

Si la brume blanche existait seule, l'emploi des filtres colorés en photographie serait inutile, pour des distances de l'ordre de io $\mathrm{km}$ ou plus faibles. En effet, pour ces distances le terme de diffusion moléculaire est petit. Soit un objet noir sur fond blanc. Admettons qu'il ne soit perceptible à distance que lorsque la différence de brillance entre l'objet et le fond du ciel est supérieure à 2 pour ıoo, et que ce chiffre soit atteint à ro $\mathrm{km}$ pour les rayons bleus $0,45 \mu)$. Un calcul simple montre que la différence des brillances, ou contraste, sera 0,026 en lumière jaune, c'est-à-dire que l'emploi d'un filtre jaune monochromatique se traduira par une augmentation de contraste de o, oo6 seulement, absolument inappréciable. Pour une limite de visibilité de ro km en lumière jaune, le contraste pour un objet noir situé à $4 \mathrm{~km}$ serait $0,23 \mathrm{I}$ (bleu) et 0,246 (jaune). Des millions d'expériences faites par les photographes attestent que les différences réelles sont beaucoup plus grandes, et que par suite la brume blanche ne peut être qu'exceptionnelle $\left(^{(}\right)$.

2. - Pour le vérifier, l'emploi de la photographie n'est pas indispensable, et il suffit d'examiner des

(1) Pour un objet vert ou jaune (feuillage) l'emploi d'un filtre vert ou jaune diminuerait les contrastes au lieu de les augmenter, c'est-à-dire que les photographes devraient au contraire employer un filtre bleu. On voit par là combien l'hypothèse de la brume blanche éloigne de la réalité. 
objets lointains au travers de filtres approximativement monochromatiques. Depuis plusieurs années, j'ai fait beaucoup d'observations de ce genre dans des lieux très divers, et dans toutes les circonstances la substitution d'un filtre jaune ou rouge à un filtre bleu a amélioré considérablement les contrastes, même à courte distance quand la diffusion moléculaire n'intervenait que pour une proportion négligeable de l'absorption. Je ne veux pas en conclure que la brume blanche n'existe pas, mais elle est si rare qu'après plusieurs années on peut très bien ne l'avoir jamais rencontrée.

En faisant les observations de très bonne heure, lorsque l'atmosphère est près du point de rosée, l'avantage du filtre jaune ou rouge est moins grand que quelques heures plus tard quand l'air s'est échauffé. Ceci montre qu'à mesure que l'atmosphère tend vers la saturation, l'absorption se rapproche de celle d'un nuage, derrière lequel le Soleil disparaît sans changer de coloration d'une manière évidente.

Je dois noter qu'en plusieurs occasions, avec une atmosphère très voisine de la saturation, les contrastes en lumière jaune ont paru plus forts qu'en lumière rouge. Il est donc possible que dans ces cas il y ait un minimum relatif d'absorption pour une longueur d'onde voisine de $0,57 \mu$.

3. Mesures. - Les faits qui précèdent sont si généraux et si frappants qu'on s'étonne qu'ils puissent être aussi souvent méconnus. Cependant on pourrait leur objecter qu'ils ne sont pas appuyés sur des nombres; qu'il y a des différences selon la couleur dans la perception des contrastes, soit par l'œil, soit par la plaque photographique; et que les objets observables sont colorés et non noirs. Soumises au calcul, ces objections se montrent sans valeur; cependant j'ai tenu à vérifier par des mesures de contraste les résultats qui précèdent.

Les vérifications sont si nettes qu'on peut employer une méthode très simple. Supposons qu'une surface noire soit observée d'un point éloigné, situé sur la même horizontale, en un lieu tel que la surface se projette sur le fond du ciel. La brillance de cette surface sera celle de la couche d'air interposée, et elle tendra vers une limite quand la distance croîtra indéfiniment, si l'éclairement de l'atmosphère reste constant sur toute cette distance. La limite sera la brillance $d u$ fond $d u$ ciel adjacent à la mire. Le contraste entre la brillance $b$ de la mire et la brillance $B$ du fond du ciel, c'est-à-dire la grandeur $B-b / B$, ne dépendra que du coefficient d'absorption de l'atmosphère. Si donc la brume est blanche, le contraste sera indépendant de la longueur d'onde, si du moins les distances sont assez courtes pour que la diffusion moléculaire n'intervienne pas.

Les contrastes ont été mesurés par photométrie photographique pour différentes longueurs d'onde isolées par des filtres. Les circonstances atmosphériques n'ont pas été favorables; alors qu'il aurait fallu des brumes denses, rapprochant la limite de visibilité à moins de $10 \mathrm{~km}$, cette limite n'a jamais été inférieure à $2 \check{5} \mathrm{~km}$ en lumière jaune Dans ces conditions, la diffusion moléculaire intervient comme correction. Pour en tenir compte, les observations sont calculées de la manière suivante :

I o On détermine le contraste pour le début de l'ultraviolet (lumière de Wood) et l'on en déduit le coefficient d'absorption correspondant à cette lumière;

$2^{\circ}$ En soustrayant le terme de diffusion moléculaire, on obtient la densité de la brume, densité indépendante de la longueur d'onde si la brume est blanche;

$3^{\circ}$ Admettant que la brume est blanche, en ajoutant à sa densité constante celle de la diffusion moléculaire pour les longueurs d'onde des différents filtres, on obtient le contraste calculé pour ces filtres;

$4^{\circ}$ Soit $\Delta$ la différence (contraste observé-contraste calculé). En cas de brume blanche et de mesures parfaites, tous les $\Delta$ devraient être nuls; par suite des erreurs de mesure, qui sont une fraction importante des quantités à mesurer, on trouvera des nombres positifs et négatifs dont la somme algébrique sera nulle ou très petite. Si au contraire la brume est bleue, toutes les longueurs d'onde utilisées étant dans le spectre visible, c'est-à-dire plus grandes que celle de la lumière de Wood, les $\Delta$ seront systématiquement positifs.

Résultats. - Les mesures ont été faites à onze dates différentes, poúr sept filtres laissant passer les longueurs d'onde o,37, o,42, o, 45, o,48, o,54, $0,58,0,65 \mu$. Je ne donnerai pas le détail des résultats obtenus, mais seulement le résultat d'ensemble.

I ${ }^{\circ}$ Nombres bruts. - Sur 53 différences :

$$
\begin{array}{ll}
31 \text { sont positives } & \text { Somme } 1,86 \\
22 \text { sont négatives } & \text { Somme } 0,92
\end{array}
$$

Ainsi les nombres positifs l'emportent du double sur les négatifs; on doit en conclure que la brume blanche, si elle existe, n'est en tout cas pas la seule : ce qui suffit à ruiner le mode de décomposition incriminé.

$2^{0}$ Nombres corrigés. - Cette première conclusion est rendue incomparablement plus nette par un examen plus attentif. Pour des études de ce genre, une mire noire artificielle ne peut être employée, car il faudrait lui donner des dimensions prohibitives (de l'ordre de ro m). Il faut donc, comme dans toutes les études de visibilité qui font partie du programme des météorologistes, utiliser les mires naturelles : je me suis servi d'un rideau de feuillage vert. J'ai déjà montré $[\mathrm{I}]$ que cette mire a un albedo faible, même dans le jaune et le vert. Sa brillance est presque négligeable vis-à-vis de celle du fond $\mathrm{du}$ ciel dans l'ultraviolet, mais elle augmente à mesure qu'on se rapproche du jaune et ne diminue 
que faiblement dans le rouge, le ciel lui-même étant peu brillant pour cette couleur. A la condition que toutes les mesures soient faites à la même heure, la brillance de la mire par rapport au ciel est peu variable et l'on peut envisager des valeurs moyennes qui sont les suivantes, en fonction de la longueur d'onde que laissent passer les filtres :

$$
\begin{array}{ccccccc}
3700 & 4200 & 4500 & 4800 & j 400 & 5,00 & 6500 \\
0,032 & 0,042 & 0,061 & 0,072 & 0,162 & 0,179 & 0,121
\end{array}
$$

Les contrastes observés seront donc inférieurs aux contrastes qu'aurait donnés une mire noire; une correction facile permet de passer des uns aux autres. Nous continuerons à appeler contrastes observés les nombres ainsi corrigés. La comparaison des contrastes observés et calculés, faite comme il est dit au paragraphe précédent, donne les résultats suivants :

$$
\begin{aligned}
& 42 \text { différences positives Somme 3,39 Moyenne o,08o } \\
& \text { Io " négatives " } 0,37 \text { ” } 0,037
\end{aligned}
$$

La somme des valeurs positives est maintenant neuf fois plus grande que celle des négatives, celles-ci étant d'ailleurs en moyenne de l'ordre des erreurs possibles.

On peut en conclure que la brume blanche, en supposant qu'elle existe, est en tout cas exceptionnelle. Normalement la brume est bleue, comme les expériences qualitatives le faisaient prévoir.

Mesures en lumière bleue. - Les nombres obtenus pour le filtre bleu $(0,45 \mu)$ sont anormaux. Pour toutes les autres couleurs, le contraste observé est en moyenne, après correction de brillance de mire, notablement supérieur au contraste calculé. Au contraire pour le bleu la différence moyenne est négligeable.

$4200 \quad 4500 \quad 4800 \quad 5400 \quad j-00 \quad 6500$ $\Delta$ moyen... $0,035 \quad 0,0050,066 \quad 0,085 \quad 0,11 \% \quad 0,107$

Il y aurait donc, un excès d'absorption dans le bleu. Il est remarquable que je suis déjà arrivé à la même conclusion dans une étude précédente [I] de telle sorte que l'existence de cet excès d'absorption est maintenant appuyée par deux séries d'expériences indépendantes.

J'avais attribué cet excès à la présence possible du peroxyde d'azote. J'ai su depuis que Reynolds [3] avait effectivement constaté la présence de peroxyde d'azote et en avait fait le dosage; il a été retrouvé par Paneth et Edgar [4]. Mon hypothèse est donc confirmée, au moins qualitativement. Les quantités trouvées sont en général inférieures à celles que j'avais envisagées (par mètre cube, o,o3 mg maximum au lieu de $0,02 \mathrm{mg}$ moyenne), mais comme j'avais reconnu la très grande variabilité du $\mathrm{NO}_{2}$ il n'y a pas pour le moment contradiction entre ces chiffres, qui sont du même ordre.

Si du tableau des différences $\Delta$ nous éliminons, comme nous avons le droit de le faire, celles qui sont relatives à la lumière bleue, l'inexistence de la brume blanche devient encore plus nette :

$$
\begin{array}{ccccc}
32 \text { différences positives donnent pour somme... } & 3,10 \\
3 \# \text { négatives " } & \# & \ldots & 0,03
\end{array}
$$

4. Loi réelle de !'absorption de la brume. Bien que les̄ expériences qui précèdent n'aient pas été conduites comme des mesures de précision, on peut en tirer une valeur moyenne des propriétés optiques de la brume dans les conditions de ces expériences. Les valeurs moyennes des contrastes, corrigées pour la brillance propre de la mire, sont :

$\begin{array}{ccccccc}3700 & 4160 & 4540 & 4700 & 5400 & 5700 & 6500 \\ 0,395 & 0,524 & 0,547 & 0,605 & 0,679 & 0,699 & 0,746\end{array}$

La distance de la mire, réduite à la densité normale de l'atmosphère, est $7300 \mathrm{~m}$. Si d'après ces nombres on calcule les coefficients d'absorption, et si l'on en retranche les valeurs données par la formule de Rayleigh (considérées comme une première approximation grossière), on obtient les coefficients d'absorption $\mathrm{K}$ de la brume moyenne. Ces coefficients sont à peu près en fonction inverse de la longueur d'onde, car le produit $\mathrm{K}^{-1}$ est à peu près constant :

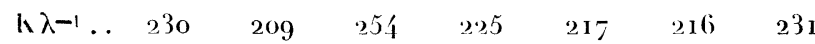

Nous retrouvons dans ces chiffres le supplément d'absorption spécifique pour le bleu, déjà signalé.

5. Brume de surface et brume d'altitude. Ces mesures ont été faites en août et septembre, dans un pays entièrement couvert de végétation et à l'abri des fumées. La brume n'était pas épaisse, puisque la limite de visibilité en jaune était comprise entre 25 et $120 \mathrm{~km}$. Elle était nettement bleue, comme on vient de le voir.

Si la brume de surface est bleue, rien n'autorise à penser que la brume d'altitude soit blanche. Il faudrait admettre que les particules qui la forment sont plus grosses que celles qui existent près du sol. Cette hypothèse demanderait une preuve formelle, sans laquelle elle est inacceptable.

Quand cette preuve n'est pas donnée, nous avons le droit de dire que la décomposition de l'absorption atmosphérique à grande altitude, en un terme de diffusion moléculaire et un terme indépendant de la longueur d'onde, est un artifice qui ne correspond pas au phénomène réel, et que les conséquences tirées de ce mode de décomposition sont sans valeur.

Manuscrit reçu le io juin 1939.

\section{BIBLIOGRAPHIE}

[1] J. Duclaux, J. de Physique, 1935, 6, p. for. - [2] J. Duclaux, J. de Physique, 193y, 10, p. 367. - [3] Rexnolds, Nature, 1938, 142, p. $571 ;$ J. Soc. Chem. Ind., 1930, 168 T. - ['́f] Paneth et Edgar, Nature, 1938, 142, p. 112 et 571 . 